\section{Discussion}

Our findings indicate, firstly, that a therapeutic degree of anticoagulation can be maintained even though hormonal contraceptives are being administered simultaneously, and, secondly, that oral contraceptives seem to potentiate the action of oral anticoagulants, although this effect is not clinically significant, and slight adjustments in the anticoagulant dose are enough to maintain prothrombin activity within the therapeutic range.

Our second conclusion does not agree with the generally accepted belief that oral contraceptives would diminish the effects of oral anticoagulants. ${ }^{3}$ This has been thought to occur through procoagulant action at three levels: (a) increased concentrations of certain coagulation factors, mainly factor VII and factor $\mathrm{X}^{45} ;(b)$ diminished concentrations of antithrombin III $^{6}$; and $(c)$ a local effect on vascular endothelia. ${ }^{7}$ Nevertheless, interaction between oral anticoagulants and oral contraceptives is quite possible at other levels, some of them imperfectly understood. The synthetic oestrogens contained in oral contraceptives may produce enzymatic inhibition in the microsomes of the hepatic cells ${ }^{8}$ which would potentiate the action of anti- coagulants through retarded metabolic degradation. ${ }^{10}$ The resultant interaction may represent only the net balance of opposing actions, which will require further study and clarification.

\section{References}

${ }^{1}$ Kaplan, N M, Annual Review of Medicine, 1978, 29, 31.

2 Poller, L, Thrombosis et Diathesis Haemorrhagica, 1975, 33, 157.

${ }^{3}$ American Pharmaceutical Association, Evaluation of Drug Interactions. Washington, APA, 1976.

${ }^{4}$ Scherogie, J J, Solomon, H M, and Zieve, P D, Clinical Pharmacology and Therapeutics, 1967, 8, 670 .

${ }^{5}$ Poller, L, et al, British Medical fournal, 1969, 1, 554.

6 Bounaemeaux, H, et al, Thrombosis et Diathesis Haemorrhagica, 1978, 39, 607.

7 Srinivasan, S, et al, Contraception, 1974, 9, 291.

${ }^{8}$ Klatskin, G, Diseases of the Liver. Philadelphia, Lippincott, 1975.

9 Song, C S, and Kappas, A, Diseases of the Liver. Philadelphia, Lippincott, 1975.

${ }^{10}$ Koch-Weser, J, and Sellers, E M, New England fournal of Medicine, $1971,285,547$.

(Accepted 29 August 1979)

\title{
SHORT REPORTS
}

\section{Survival after bilateral renal artery occlusion}

Embolic occlusion of a renal artery is uncommon and the clinical diagnosis made rarely. We report a case of sequential bilateral renal emboli successfully treated conservatively without surgical intervention.

\section{Case report}

A 64-year-old man with a history of hypertension and ischaemic heart disease was admitted with a two-day history of right-sided abdominal pain and vomiting. He had atrial fibrillation, a blood pressure of $160 / 80 \mathrm{~mm} \mathrm{Hg}$, and tenderness in the right loin. An intravenous urogram (IVU) showed a non-functioning right kidney. Several hours after admission he complained of a cold, rumb right arm with absent arterial pulsation distal to the axillary artery. Right brachial embolectomy was performed under local anaesthesia. After this a renal arteriogram showed emboli in the right artery (figure). The left renal arterial tree was normal. Anticoagulant treatment with heparin was given and continued after discharge by subcutaneous self-administration (7500 units eight hourly). An oral anticoagulant was not used because of a history of heavy alcohol consumption and abnormal liver-function test results. One month later the patient was readmitted with anuria and complaining of severe left-sided abdominal pain. An IVU showed no function in either kidney. A renal arteriogram showed a patent right renal artery, though some branches were occluded, and much reduced perfusion of the left kidney, suggesting gross infarction. Streptokinase $100000 \mathrm{U}$ was injected into the left renal artery. Forty-eight hours later he was still anuric with severe hyperkalaemia and a metabolic acidosis. Serum creatinine concentration was $1110 \mu \mathrm{mol} / 1(12.5 \mathrm{mg} / 100 \mathrm{ml})$. Peritoneal dialysis was started together with intravenous heparin 10000 units six hourly. Seven days later he began to pass urine, and by day 14 his renal function was sufficient to discontinue dialysis (serum creatinine concentration $170 \mu \mathrm{mol} / 1(1.9 \mathrm{mg} /$ $100 \mathrm{ml})$ ). He was discharged home on warfarin. A subsequent IVU showed recovery of function in both kidneys.

\section{Comment}

The diagnosis of renal artery embolism is often delayed because its clinical features are common to other conditions. In this case the right brachial artery embolus led to early diagnosis of the first episode. Previously reported cases have shown that kidney function may return despite prolonged interruption of blood flow. Some protection may be given by the small collateral venovenous anastomoses from the ureter, the capsule, and the perinephric tissues. ${ }^{1}$ The renal artery pressure may be inadequate for filtration but at $25 \mathrm{~mm} \mathrm{Hg}$ will still maintain renal integrity. ${ }^{2}$ Other possible explanations for recovery of renal function include spontaneous fibrinolysis, recanalisation of the artery, or intense vasospasm associated with occlusion of a segmental branch of the renal artery with vasoconstriction producing a syndrome similar to acute tubular necrosis. ${ }^{3}$ The duration of the renal failure in our patient favours the latter explanation, and segmental occlusion of a renal artery is known to cause complete non-visualisation on an IVU. ${ }^{4}$

We believe that this is the first reported case of bilateral sequential renal emboli with survival after medical treatment alone. Moyer et al $l^{5}$ reviewed confirmed reports of renal artery embolism over the past 20 years and found 39 cases, of which 24 were treated surgically with six deaths and 15 were treated medically with two deaths. Renal function recovered in 10 of the survivors treated medically and in seven treated surgically. The success of medical treatment in this condition questions
Renal arteriogram showing embolisation of right renal arterial tree. Normal left renal arterial circulation. 
the indications for surgical intervention, especially when many of these patients have ischaemic or rheumatic heart disease.

${ }^{1}$ Smith, G T, fournal of Urology, 1963, 89, 275.

${ }^{3}$ Halpern, M, Fournal of Urology, 1967, 98, 552.

4 Siegelman, S S, and Goldman, A G, Radiology, 1968, 90, 1084.

${ }^{5}$ Moyer, J D, fournal of Urology, 1973, 109, 138.

(Accepted 4 September 1979)

\section{Royal Sussex County Hospital, Brighton BN2 5BE}

R J S MCGONIGLE, MB, MRCP, medical registrar

J A P TRAFFORD, FRCP, consultant physician and director of renal unit P SHARPSTONE, FRCP, consultant physician

J S TAPSON, MB, senior house officer
2 Morris, G C, Heider, C F, and Moyer, J H, Surgical Forum, 1955, 6, 623.

was supple. The scalp was hyperalgesic over the left temporo-occipital region. His motor and sensory functions were normal. $\mathrm{He}$ walked on a broad base. Plantar response was flexor and deep tendon reflexes normal. His urine and blood chemistry were normal. The absolute eosinophil count was $3.8 \times 10^{9} / 1\left(3800 / \mathrm{mm}^{3}\right)$. ESR was $35 \mathrm{~mm}$ in $1 \mathrm{st} \mathrm{h}$ and the coagulation profile normal. No haemoparasites were seen. Stool examination showed no parasites or ova. The bone marrow showed hypercellularity with $15 \%$ eosinophils. Antinuclear antibody and LE-cell tests were negative. Filarial complement-fixing antibody test was positive. Spinal fluid was normal. Skull radiographs were normal. Arch angiogram showed no abnormality except congenital absence of the right posterior inferior cerebellar artery. Amipaque (metrizamide) ventriculogram was also normal. On treatment with prednisolone and diethylcarbamazine the scalp hyperalgesia, ataxia, and nystagmus improved. Repeat haemogram on day 14 showed an absolute eosinophil count of $1.45 \times 10^{9} / 1\left(1450 / \mathrm{mm}^{3}\right)$. By three weeks the ataxia had disappeared completely. Diethylcarbamazine was continued for 21 days and review after two months showed no neurological deficit.

\section{Comment}

Tropical eosinophilia is a hypersensitivity response to an exciting allergen invariably parasitic, the commonest being filaria. ${ }^{2}$ Neurological manifestations are very rare, in contrast to hypereosinophilic syndromes associated with severe vasculitis like polyarteritis nodosa and Wegener's granulomatosis. The patients presented here had definite eosinophilia, fulfilling all the criteria of the hypereosinophilic syndrome. ${ }^{3}$ With ascariasis excluded by stool examination, it is postulated that both patients had the eosinophilic syndrome due to filariasis. The positive filarial complement fixation test was confirmatory. Neither patient had a detectable neurological abnormality in spite of signs of focal disease. This, in combination with a rapid recovery, suggested a reversible disorder like a benign vasculitis or eosinophilic infiltration.

${ }^{1}$ Lancet, 1977, 1, 943.

${ }^{2}$ Udwadia, F E, Fournal of the Association of Physicians of India, 1978, 26, 429.

${ }^{3}$ Udwadia, F E, in Progress in Respiration Research, vol. 7. Basel, S Karger, 1975.

(Accepted 29 August 1979) memory 37 -year-old right-handed housewife developed impairsent right half of her body. In the beginning she had cough, chest pain, and breathing difficulty. Within a week she became disorientated and started behaving abnormally, like undressing in front of her family. She had no fever, visual disturbances, weakness, or convulsions. She was well developed. System review was normal. Her memory was defective, especially for recent events, and she could not do even simple arithmetic. Speech showed anomic aphasia. Her writing was grossly abnormal with omission of letters and loss of spatial organisation. She also showed finger agnosia, right-left disorientation, and constructional difficulty. The cranial nerves were intact and optic fundi normal. There was no disturbance of motor function, but cortical sensory loss on the right side was conspicuous. The deep tendon reflexes were normal and plantar response flexor. Results of urine and blood analysis were normal. ESR was $68 \mathrm{~mm}$ in $1 \mathrm{st} \mathrm{h}$. She had an absolute eosinophil count of $11.5 \times 10^{9} / 1\left(11500 / \mathrm{mm}^{3}\right)($ table). Serum protein electrophoresis

Haemogram in case (case 1) of tropical eosinophilia

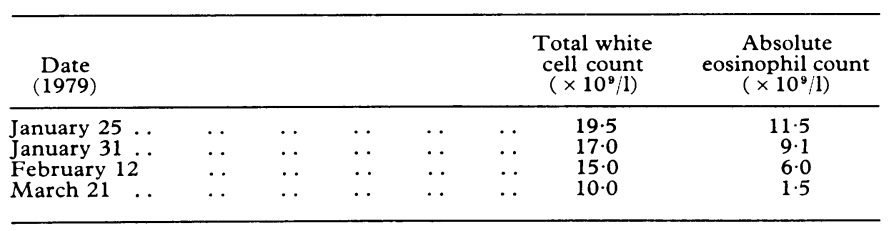

Conversion: SI to traditional units-Blood cells: $1 \times 10^{9} / 1 \approx 1000 / \mathrm{mm}^{3}$.

was normal and LE cells were absent. No haemoparasites were seen but filarial complement-fixing antibody test was positive. The venereal disease research laboratory test was negative and coagulation profile normal. Bone marrow showed eosinophilic hyperplasia. Spinal fluid was normal. Stool showed a few ova of Ancylostoma duodenale. Skull and chest radiographs and ECG were normal. An electroencephalogram was mildly and diffusely abnormal with slow waves of 6-7 cps. Left carotid angiogram was normal. Treated with diethylcarbamazine $100 \mathrm{mg}$ thrice daily she improved within two weeks. Her numbness disappeared first, then her finger agnosia and right-left disorientation. Her writing was the last to recover. Eight weeks later the patient had recovered completely.

(2) A 9-year-old boy was admitted with a two-month history of persistent, severe left temporo-occipital scalp tenderness. One week before he had developed double vision and loss of balance, controlled by closing his left eye. He had no fever, cough, chest discomfort, or convulsions. System review was normal. Mental functions, speech, visual fields, optic fundi, and pupils were normal. He kept his left eye constantly closed. On opening it he became ataxic. There was vertical and horizontal nystagmus and central facial paralysis of the right side. Other cranial nerves were intact. The neck

Department of Neurology, Sree Chitra Tribunal Medical Centre, Trivandrum, $\mathbf{S}$ India

M RAVINDRAN, MD, FRCPED, professor and head of department

\section{Cervical myelopathy after metrizamide myelography}

Metrizamide (Amipaque), a water-soluble contrast medium, is increasingly being used for cervical and thoracic myelography as well as lumbar myelography. It differs from other water-soluble contrast media in being non-ionic, and side effects of metrizamide occur less often and are less severe than with other agents. ${ }^{1}$ We report a case of cervical myelitis occurring after lumbar myelography with metrizamide.

\section{Case report}

A 29-year-old man presented to the orthopaedic department of a peripheral hospital with a one-year history of low backache exacerbated by lifting heavy weights. Examination showed limited movement of the lumbar spine, with bilateral reduction of straight-leg raising. Neurological findings were normal. Lumbar myelography was performed with $10 \mathrm{ml}$ metrizamide introduced atraumatically at the L3-4 level. The spinal canal was narrowed but no disc protrusion was seen. Within minutes he developed severe headache associated with paraesthesiae in the hands and back of the legs. These symptoms resolved after about 30 minutes, but he noticed flaccid weakness of the left forearm and hand. He was transferred to Nottingham General Hospital.

Examination confirmed weakness of all muscle groups below the elbow in the left arm, predominantly of a C8-D1 distribution, with associated hyperreflexia. The right arm was normal. Abdominal reflexes were symmetrical, and there were no motor signs in the legs. Sensory examination disclosed subjective impairment of pain sensation over the outer aspect of the left arm and forearm within the $\mathrm{C} 6$ dermatome. Cranial nerves were normal. Radiography of the skull showed no abnormality at the craniovertebral junction. Cervical spine radiography showed a narrow but adequate canal. 